\title{
Tuning and Topography in an Odor Map on the Rat Olfactory Bulb
}

\author{
Markus Meister ${ }^{1,2}$ and Tobias Bonhoeffer ${ }^{2}$ \\ ${ }^{1}$ Harvard University, Cambridge, Massachusetts 02138, and ${ }^{2}$ Max Planck Institute of Neurobiology, D-82152 Munich- \\ Martinsried, Germany
}

\begin{abstract}
The sense of smell originates in a diverse array of receptor neurons, comprising up to 1000 different types. To understand how these parallel channels encode chemical stimuli, we recorded the responses of glomeruli in the olfactory bulbs of the anesthetized rat, by optical imaging of intrinsic signals. Odor stimulation produced two kinds of optical responses at the surface of the bulb: a broad diffuse component superposed by discrete small spots. Histology showed that the spots correspond to individual glomeruli, and that $\sim 400$ of them can be monitored in this way. Based on its wavelength-dependence, this optical signal appears to derive from changes in light scattering during neural activity. Pure odorants generally activated several glomeruli in a bilaterally symmetric pattern, whose extent varied greatly with concentration. A simple formalism for ligand binding accounts quantitatively for this con-
\end{abstract}

Our olfactory system senses chemicals in the ambient air through specialized receptor cells that are located in the neural epithelium lining the upper reaches of the nasal cavity. Each of these olfactory neurons is thought to express a single type of receptor protein that spans the plasma membrane and whose binding properties determine the interaction with extracellular ligands (Buck and Axel, 1991; Buck, 1996; Mombaerts, 1999). In this respect, olfactory receptors are similar to photoreceptors: each photoreceptor cell makes a single type of opsin protein, whose absorption properties determine the interaction of the cell with photons of different wavelength. However, photoreceptors come in only a handful of varieties, whereas mammals seem to have up to 1000 types of olfactory neurons (Mombaerts, 1999). Therefore, fully $1 \%$ of our genome may be dedicated to generating the diversity of olfactory receptors. In this great variety of primary receptors, the olfactory system is unique compared with all other senses. How is sensory information processed in a system that from the very outset uses such a large number of parallel channels?

The number of distinct organic structures that elicit a smell in humans is clearly much larger than the number of olfactory receptor genes (Mori et al., 1998). Therefore, many of the $\sim 1000$ receptors must be promiscuous and bind to several different ligands. In fact, physiological experiments have shown that a given olfactory neuron can often be stimulated by many com-

\footnotetext{
Received Sept. 15, 2000; revised Dec. 4, 2000; accepted Dec. 5, 2000.

M.M. was supported by grants from the Office of Naval Research (N00014-98-10829) and the National Science Foundation (IBN 9453317), and T.B. was supported by the Max Planck Gesellschaft. We thank Imke Goedecke, Iris Kehrer, and Frank Sengpiel for experimental advice and assistance and Catherine Dulac and members of our laboratories for comments on this manuscript.

Correspondence should be addressed to Markus Meister, Harvard University, 16 Divinity Avenue, Cambridge, MA 02138. E-mail: meister@biosun.harvard.edu. Copyright (C) 2001 Society for Neuroscience $0270-6474 / 01 / 211351-10 \$ 15.00 / 0$
}

centration dependence and yields the effective affinity with which a glomerulus responds to an odorant. When tested with aliphatic molecules of increasing carbon chain length, many glomeruli were sharply tuned for one or two adjacent chain lengths. Glomeruli with similar tuning properties were located near each other, producing a systematic map of molecular chain length on the surface of the olfactory bulb. Given local inhibitory circuits within the olfactory bulb, this can account for the observed functional inhibition between related odors. We explore several parallels to the function and architecture of the visual system that help interpret the neural representation of odors.

Key words: olfaction; receptor; glomerulus; map; tuning; imaging

pounds, even structurally very dissimilar molecules (Gesteland et al., 1963; Duchamp et al., 1974; Sicard and Holley, 1984; Duchamp-Viret et al., 1999). For technical reasons, these experiments used a limited set of stimuli, sometimes at rather high odor concentrations, to ensure that responses could be detected at all. Thus, it remains unclear for any olfactory receptor what the full range of active ligands is, whether there is one with specially high affinity, and which ligand is dominant under natural conditions of stimulation. Still, it is safe to assume that individual odorantsand certainly the mixtures of compounds that characterize real olfactory objects-generate a pattern of activity across several receptors. To understand this code, it would obviously be useful to monitor in parallel the responses of many receptor types.

Among the tens of millions of neurons in the olfactory epithelium, the different receptor types are widely interspersed: each type is restricted to one of four coarse zones of expression, but there is no apparent order within each zone (Buck, 1996). A receptor cell projects its axon to the olfactory bulb, where it terminates in a small ball of neuropil, a glomerulus. The rat olfactory bulb has $\sim 2400$ of these glomeruli, close-packed in a layer just beneath the surface (Meisami and Sendera, 1993). Olfactory neurons of a given receptor type send their axons to just two glomeruli in the bulb (Ressler et al., 1994; Vassar et al., 1994; Mombaerts et al., 1996a). Given that there are approximately twice as many glomeruli as receptor types, it is plausible that each glomerulus receives axons from just one receptor type, although direct evidence on this point is still lacking (Mombaerts, 1999). If so, then the olfactory bulb effectively acts as a switchboard, collecting the signals of a given receptor type from a broad zone of the epithelium into a small brain region $\sim 150 \mu \mathrm{m}$ in diameter. The structure of this switchboard is stereotyped and precise: glomeruli for the various receptor types are generally 
located at mirror-symmetric positions in the two bulbs, and their arrangement is reproducible across individuals (Ressler et al., 1994; Vassar et al., 1994; Mombaerts et al., 1996b). Thus, the projection to the olfactory bulb maps every possible odor into a two-dimensional image of neural activity in the layer of glomeruli.

These neural images elicited by odors on the olfactory bulb have been a subject of great interest (Shepherd, 1994; Xu et al., 2000). Early experiments showed that odorants with very different chemical properties also produce different activity patterns (Stewart et al., 1979; Jourdan et al., 1980; Cinelli et al., 1995). More importantly, molecules with similar chemistry tend to produce related patterns. Certain regions of the bulb appear to be dedicated to odorants of a particular chemical class (Imamura et al., 1992; Katoh et al., 1993; Johnson et al., 1999; Johnson and Leon, 2000). Odorant molecules with several different functional groups may activate several regions in a combinatorial manner (Friedrich and Korsching, 1997; Johnson et al., 1998). Collectively, these studies suggest that there is a coarse topography of chemical properties on the surface of the bulb, although the rules of that map remain to be discovered. Many recent contributions in this area relied on the method of 2-deoxyglucose labeling, which marks cells in the brain that have increased metabolic activity during exposure to an odor. A great boon of this technique is that it allows observation of all glomeruli simultaneously, and thus gives access to the entire odor-evoked neural image. A drawback is that one can inspect the image for only one odor at one concentration per experimental animal. Although it is possible to align the patterns from different animals by anatomical landmarks on the bulb, the interindividual differences in glomerular arrangements have limited the spatial resolution of the resulting odor maps (Johnson and Leon, 2000).

As demonstrated recently (Rubin and Katz, 1999; Uchida et al., 2000), one can also observe odor maps directly by optical recording of so-called "intrinsic signals" from the surface of the olfactory bulb. Here we use this method to monitor simultaneously $\sim 200$ glomeruli in each olfactory bulb of an anesthetized rat stimulated with air-borne odor pulses. To document the foundations of the technique, we investigate the origin of the optical signals and demonstrate that they can reliably resolve neighboring glomeruli. We then take advantage of the ability to deliver many odors to study the chemical tuning of the glomeruli, in particular their sensitivity to the size of an odor molecule. Individual glomeruli can be rather sharply tuned along this size axis, but the tuning curve broadens at high odor concentration. We find a quantitative explanation for this behavior by measuring the effective affinity of the glomerulus for each odor. This affinity spectrum determines how the glomerulus participates in different odor maps. Finally, we analyze how the glomeruli with different spectra are arranged on the surface of the olfactory bulb. We show that there is fine structure in this map on the scale of a few interglomerular distances: nearby glomeruli systematically have similar response properties.

\section{MATERIALS AND METHODS}

Preparation. Six adult rats (female Wistar, $\sim 200 \mathrm{gm}$ ) were used for the imaging experiments reported here. They were anesthetized with urethane $(12.5 \%$, i.p., $2.5 \mathrm{ml}$ initial dose) and mounted in a stereotaxic frame. A hole was cut in the skull revealing the dorsal surface of both olfactory bulbs (on the midline, 5-mm-wide, from 4 to $11 \mathrm{~mm}$ anterior to the bregma). The dura was left intact, and the opening was filled with transparent agar (Biomol, Hamburg, Germany), and covered with a glass coverslip, to reduce movement of the brain. A solution of nutrients was injected under the skin of the neck ( $2 \mathrm{ml}$ of amynin; Rhone Merieux, Athens, GA). Heart beat, respiratory rate, and lack of pain reflexes were monitored continuously. All animal protocols conformed to National Insti- tutes of Health guidelines and were approved by Harvard University's Animal Care and Use Committee, as well as the Regierung von Oberbayern.

Stimulation. Odors were delivered from a stimulator with eight tanks containing different odorants (AutoMate Scientific, San Francisco, CA). Humidified air passed through a tank containing filter paper soaked with odorant diluted in mineral oil. This air was diluted fivefold with clean air and directed at the nose with a final flow rate of $2500 \mathrm{ml} / \mathrm{min}$. A four-way valve close to the nose switched between this stream of odorized air and a stream of clean air. The animal breathed through the nose at a stable rate of $\sim 2$ inspirations/sec.

The odor concentration was controlled by varying the $\mathrm{v} / \mathrm{v}$ dilution of odorant in mineral oil, $D$, over the range from 0.0001 to 0.1 . The final vapor concentration was measured with a flame ionization detector (MicroFID; Pine Environmental, Cranbury, NJ), and found to be approximately proportional to the dilution ratio $D$. To obtain the odor concentration as a fraction of saturated vapor, for the aldehyde with $L$ carbons at dilution $D$, multiply $D$ by $0.063(L=4), 0.12(L=5), 0.24(L=$ 6), $0.47(L=7), 0.93(L=8)$, or $1.8(L=9)$.

The data acquisition computer controlled the eight valves to select the active odor tank and the four-way valve that determined onset and offset of odor stimulation. Generally, a 12 sec odor exposure was followed by 12 sec of clean air, followed by the next odor. The sequence of presentations was pseudorandom, ensuring that each odor was presented exactly once in a cycle. One of the tanks of the stimulator was left empty, providing a control condition of stimulation without odorant.

Imaging. Images of the brain surface were recorded through the agar window as described elsewhere (Bonhoeffer and Grinvald, 1996). In brief, the brain was illuminated with $707 \mathrm{~nm}$ light using a stable incandescent source and an interference filter ( $20 \mathrm{~nm}$ pass band). A cooled CCD camera (OR A 2001; Optical Imaging, Germantown, NY) equipped with two front-to-front photo objectives recorded images (resolution $192 \times 144)$ in $0.5 \mathrm{sec}$ frames. Acquisition began $3 \mathrm{sec}$ before odor onset and ended $12 \mathrm{sec}$ after odor onset (Fig. 1). At periodic intervals, a few images were recorded at $546 \mathrm{~nm}$ wavelength, to visualize the pattern of blood vessels on the surface of the olfactory bulb. This served to adjust the camera focus, which was nominally $400 \mu \mathrm{m}$ below the surface vessels.

To obtain the odor-induced absorption change, we divided the image recorded during odor stimulation by the image just preceding odor onset. This ratio image was low-pass-filtered by convolution with a Gaussian spatial kernel (SD $175 \mu \mathrm{m}$ ). High-pass filtering was achieved by subtracting the low-pass-filtered version from the raw ratio image (Fig. 1). Note that the discrete spots identified in these images are considerably smaller than the Gaussian kernel (Fig. 2), and thus their shape is not affected by the spatial filtering. With strong stimulation, the odor image was discernible on single trials. To improve the signal-to-noise ratio, the images reported here were averaged over 16-24 trials with the same odorant, interleaved with other odorants over the course of $\sim 1 \mathrm{hr}$. Odor images at the beginning and end of this period were consistent, but gradually declined in amplitude, typically by $\sim 1 / 3$. After a rest period without stimulation, the response amplitude recovered.

Image analysis. To measure the size and amplitude of a given spot in the ratio image, the spot profile was fit by a two-dimensional Gaussian. When the strength of the same spot was compared in different odor images, the center and width of the Gaussian were obtained from the strongest image, and the amplitude was adjusted for each of the other images to provide the best fit. Responses are quoted as the relative increase in absorbance at the center of the spot. The preferred chain length $<\mathrm{L}>$ of a glomerulus was computed by weighting the carbon numbers of the odorants with their respective responses,

$$
\langle L\rangle=\sum_{L=4}^{9} L \cdot R_{L} \mid \sum_{L=4}^{9} R_{L},
$$

where $L=4, \ldots, 9$ is the aldehyde carbon chain length, and $R_{L}$ the corresponding response. Note that $\langle\mathrm{L}\rangle$ can take on noninteger values. For this purpose, the responses were measured at intermediate concentrations $(D=0.01)$.

Histology. A rat was perfused through the heart with saline followed by $4 \%$ paraformaldehyde in phosphate buffer. The brain was removed and post-fixed for $2 \mathrm{hr}$, then transferred to $30 \%$ sucrose overnight. The olfactory bulbs were frozen and cut into $40 \mu \mathrm{m}$ sections, either horizontal or parasagittal. Alternating sections were mounted and dried on gelatin slides. One set of sections was stained with cresyl violet, the intervening ones were reacted for cytochrome oxidase (Wong-Riley, 1979).

These procedures led to tissue shrinkage by a factor of 1.4 , judged by measuring the overall dimensions of the bulb before and after processing. 

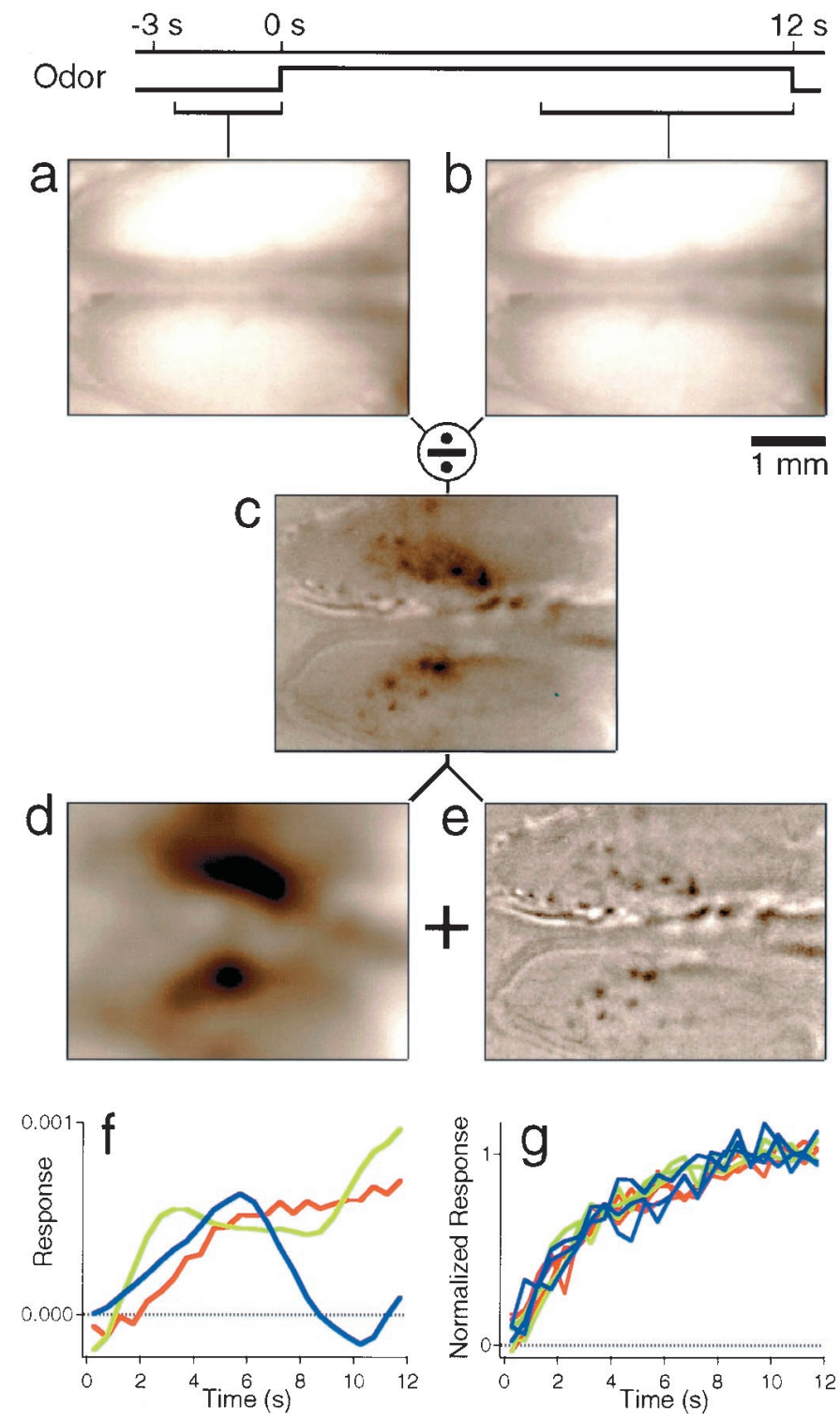

Figure 1. Local and diff use components of intrinsic images. A reference image $(a)$ is taken over a $2.5 \mathrm{sec}$ period before odor stimulation and another image $(b)$ over a $6 \mathrm{sec}$ period during stimulation (with valeraldehyde at concentration $D=0.01$; see Fig. 3 ). The ratio of the two images (c) reveals absorbance changes of the tissue. This ratio image can be decomposed into components of low $(d)$ and high $(e)$ spatial frequency. The low-frequency component $(d)$ was obtained by convolution with a Gaussian kernel of $0.175 \mathrm{~mm}$ SD. Subtracting this from the raw image yields the high-frequency component $(e)$. The gray scale in $e$ ranges from 0.9993 to 1.0007 . In these and all subsequent images, the anterior end of the bulb is on the left. $f$, Time course of the low-frequency component after onset of stimulation with this odor, in three different animals. $g$, Time course of the local component, measured for three spots in each of three animals. Because the response amplitudes vary among different spots, they were normalized to the same plateau value.

To determine the dimensions of glomeruli in the dorsal bulb, several adjacent sections were overlaid and aligned. Each glomerulus was measured in the section where it had the largest cross-sectional area. Areas quoted in Results are corrected for shrinkage and correspond to the clear region in Nissl stains, which is bordered by cell bodies of periglomerular and glial cells.

\section{RESULTS}

\section{Diffuse and local signals in intrinsic images}

The dorsal surface of both olfactory bulbs was imaged in deep red light, focusing $\sim 400 \mu \mathrm{m}$ below the superficial blood vessels (see
Materials and Methods). Stimulation with certain odors produced small but distinct optical changes in the image, amounting to a decrease in reflectance of a few parts in 1000 (Fig. 1). In general, this signal contained two components: a diffuse dark smear superposed by small dark spots. The two can be separated by applying a low-pass spatial filter to the raw image; after subtracting the filtered image from the original image, the residual image contains only the discrete spots (Fig. 1e).

To test whether these two components have different physiological significance, we inspected their time course after odor onset (Fig. 1f,g). The discrete spot signal had a very stereotyped time course: it rose immediately after odor onset with a half-time of $\sim 2-3 \mathrm{sec}$, then it saturated (Fig. $1 g$ ). This time course was similar for different spots and also across animals. By contrast, the response of the diffuse component varied greatly across animals and even between presentations of the same odor (Fig. 1f). The diffuse signal also contained a pronounced oscillatory component, with a temporal period of $\sim 10 \mathrm{sec}$, which was observed even without odor stimulation (data not shown). This may originate in slow oscillations of the cerebral vasculature (Mayhew et al., 1996). The diff use signal was generally stronger than the small spots, on occasion up to 10 -fold, but because the two components differ so much in spatial scale, the above filtering procedure isolated the local spots very effectively.

\section{Local signals originate in glomeruli}

After odor stimulation, the first neural event in the olfactory bulb is the activation of afferent terminals localized in the glomeruli. Thus, it is tempting to propose that the discrete spot-like signals reflect neural activity in individual glomeruli. To test this further, we compared the size and shapes of anatomical glomeruli with those of spots in the optical responses. Figure $2 a$ shows a horizontal section through the dorsal olfactory bulb, stained to reveal cytochrome oxidase activity. The neuropil of glomeruli is stained darkly, whereas the boundary regions between them are pale. Because the dorsal surface is curved, a series of subsequent sections was used to reconstruct it, yielding the cross sections of 200 glomeruli that could be viewed by imaging from the dorsal side (Fig. 2a). They range considerably in size (Meisami and Sendera, 1993), approximately a factor of two in linear dimension, and also in the degree of elongation (Fig. 2b). For comparison, we measured the intensity profile of discrete spots (as in Fig. 1e) observed in optical images from several experiments. These spots matched the glomeruli very well in the distributions of both size and shape (Fig. 2b). Because there is no other anatomical structure in the superficial bulb with these same properties, it appears very likely that the discrete spots correspond to activated glomeruli.

In vertical histological sections (data not shown), the glomeruli appear as slightly flattened spheroids. If the optical signal originated entirely within the glomerulus and was uniformly distributed there, the intensity profile of the dark spots should also have a spheroid shape. Instead, we found that the typical spot profile was much better described by a Gaussian. Comparing the profiles of the average glomerulus and the average spot (Fig. $2 b$ ), one finds that the optical signal extends only slightly beyond the anatomical dimension of a glomerulus and falls off rapidly within $50 \mu \mathrm{m}$. It is undetectable one glomerular diameter away. This shows that the spots represent individual glomeruli, not pairs or small clusters, and that neighboring glomeruli can be resolved reliably. For further analysis of odorant responses, we therefore focused exclusively on the early spot-like signals. 
a

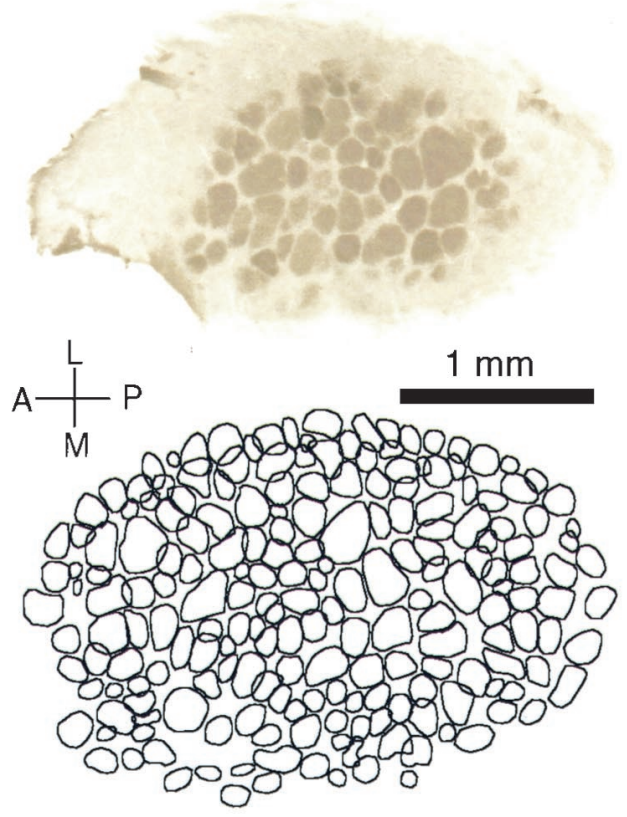

b
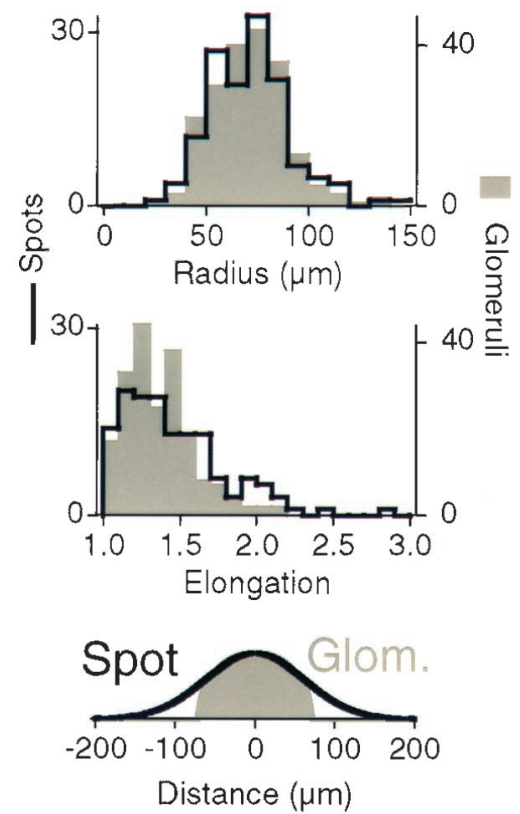

C

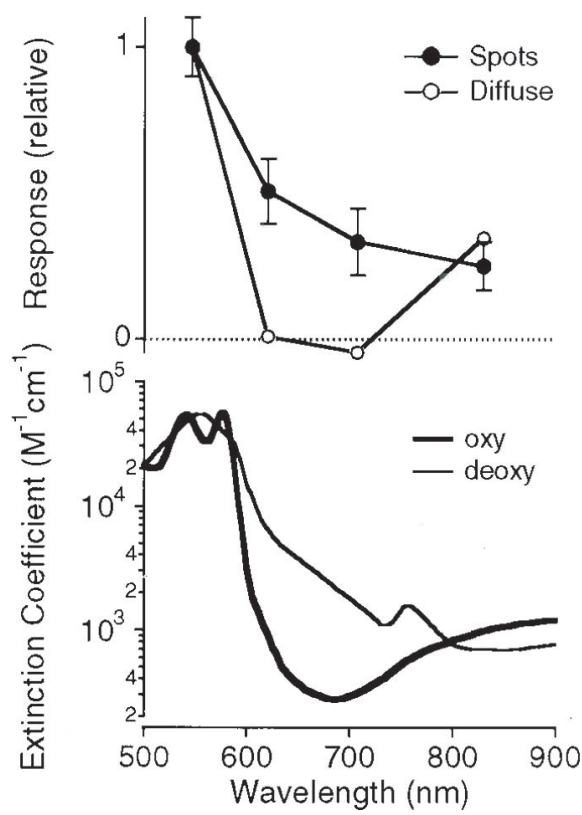

Figure 2. Origin of the spots in intrinsic images and their correspondence to glomeruli. $a$, Horizontal section (top) through the dorsal glomerular layer of the right olfactory bulb of a rat, stained by the cytochrome oxidase reaction. To reconstruct all dorsal glomeruli accessible to optical recording, nine successive horizontal sections were aligned. For each of 200 glomeruli in these sections, the largest profile in the series was drawn (bottom). Note some stacking of glomeruli near the top of the image, where the lateral wall of the bulb begins to curve down. Scale bar, $1 \mathrm{~mm}$, corrected for tissue shrinkage. $b$, Distribution of the radius (top) and the elongation (middle) measured for 200 glomeruli in histological sections (shaded) and also for 139 spots obtained in optical recordings of odorant responses from four rats (solid line). For a glomerulus, the radius $r$ was computed from the area of the largest horizontal cross section $(a)$ as $r=V_{\text {area }} / \pi$; the elongation, $e$, is the ratio of the longest diameter of the cross section to its width perpendicular to that line. For a spot observed in optical recordings, the intensity profile was fitted with a two-dimensional Gaussian with SDs $\sigma_{1}$ and $\sigma_{2}$ along the major and minor axes. The radius was defined as $\mathrm{R}=\sqrt{ } \ln 4 \sqrt{ } \sigma_{1} \cdot \sigma_{2}$; this is the average radius of the contour line that encloses $50 \%$ of the volume under the Gaussian. The elongation was taken as $E=\sigma_{1} / \sigma_{2}$. Bottom, The spheroid vertical profile of the average glomerulus (using $r=71 \mu \mathrm{m}$ ) is compared with the Gaussian intensity profile of the average spot $(R=72 \mu \mathrm{m}) . c$, Top, The optical response of five small spots (closed symbols, mean $\pm \mathrm{SD}$ ) and of the surrounding diffuse signal (open symbols) in the same olfactory bulb, measured at different wavelengths. All spectra are plotted relative to the signal at 546 nm. Bottom, The absorption spectra of oxyhemoglobin and deoxyhemoglobin (Takatani and Graham, 1979), plotted on a logarithmic axis.

\section{Source and spread of the optical signal}

Several biophysical sources are known to contribute to intrinsic optical signals elicited by sensory stimulation. One component of optical signals is triggered by the increased oxygen consumption from elevated neural activity. This in turn alters the oxygenation of hemoglobin in nearby microcapillaries, with consequent changes in the hemoglobin absorption spectrum. To test whether this was the main signal source here, we performed measurements at four different wavelengths: 546, 620,707, and $830 \mathrm{~nm}$. In all cases, odor stimulation produced spots of increased absorption. The strength of this spot signal decreased somewhat at longer wavelengths, although only gently (Fig. $2 c$ ). By contrast, the extinction coefficients of oxyhemoglobin and deoxyhemoglobin vary greatly over this range of the spectrum, and the two absorption spectra even cross between 707 and $830 \mathrm{~nm}$, with an isosbestic point in between (Fig. $2 c$ ). If a change in the oxygenation of hemoglobin were the dominant signal source, the optical responses should have opposite sign at these two wavelengths, counter to what we observed. Similarly, a change in total blood content seems an unlikely signal source, because hemoglobin absorption varies with wavelength much more strongly than the response. Altogether, it seems unlikely that changes in the properties of blood account for the spot-like signals measured here. An alternative explanation is that neural activity leads to small changes in cell volume, which produce altered optical scattering. This effect would have broad-band spectral characteristics, with a strength decreasing gently with wavelength (Mayhew et al., 1999; Vanzetta and Grinvald, 1999), as observed here for the spot signal. By contrast, the diff use signal in the area surrounding the spots had a stronger wavelength dependence; it even changed sign between 707 and $830 \mathrm{~nm}$, crossing the isosbestic point of hemoglobin (Fig. $2 c$ ). Qualitatively, this spectrum suggests a contribution from light scattering and also from a shift toward oxyhemoglobin. Thus, it appears likely that the spots and the diffuse signal have different origins; the latter may derive from activation of deeper layers of the bulb as well as spontaneous variations in blood flow.

Regardless of the biophysical mechanism of the optical changes, Figure $2 b$ shows that the spot signal does not "leak" far out of the activated glomerulus. The spread of $\sim 50 \mu \mathrm{m}$, which is considerably smaller than the size of a glomerulus, sets the spatial resolution limit of our optical measurements. The rise time of 2-3 sec (Fig. 1g) sets the temporal resolution of the technique: clearly this is too slow to resolve the precise onset of the neural response, or the modulation by the sniff cycle, or the fast temporal patterning of activity that emerges in the olfactory bulb (Laurent, 1999; Mori et al., 1999). As in other systems, intrinsic signal imaging can serve to inspect the spatial distribution of neural activity, not its precise temporal structure. However, the specific conclusions discussed below, regarding tuning of the odorant receptors and the spatial arrangement of their signals on the olfactory bulb, are all based on the steady-state activity at late times in the response and do not require a resolution of the detailed time course. 


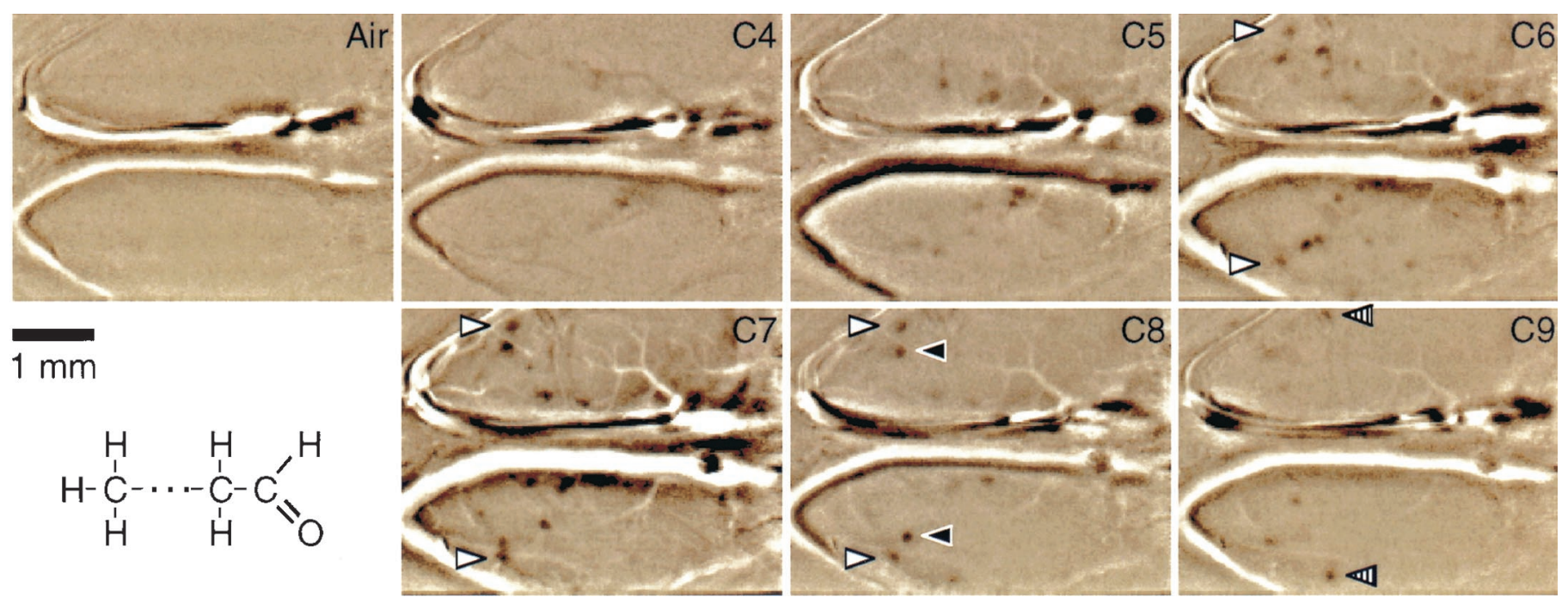

Figure 3. Optical responses to aliphatic aldehydes. The stimuli were aliphatic aldehydes of carbon chain lengths 4 to 9 , presented at a mineral oil dilution of $D=0.01$ (see Materials and Methods for corresponding vapor concentrations), and included a control with clean air. Images were averaged from 6 to $12 \mathrm{sec}$ after odor onset and divided by the image 2.5 to $0 \mathrm{sec}$ before onset. The resulting ratio images were high-pass filtered as in Figure $1 b$. The gray scales are the same in all panels and range from 0.9993 (black) to 1.0007 (white). Matching arrowheads indicate examples of active glomeruli that are clearly paired symmetrically across the midline. Some glomeruli are activated by only one odor in this series (e.g., black and striped arrowheads), others respond to several odors (e.g., white arrowheads).

\section{Responses to a series of aliphatic aldehydes}

To explore the mapping of odor properties on the surface of the olfactory bulb, we chose a series of molecules with identical functional groups and charge, but varying only in size: the saturated aliphatic aldehydes (Imamura et al., 1992). Figure 3 illustrates the optical response to different odors in this series, whose carbon number ranged from 4 (butanal, abbreviated "C4") to 9 (nonanal, "C9"). A control experiment using clean air produced a flat ratio image, except for movement artifacts along the boundaries of the two olfactory bulbs. Images obtained with odor stimulation showed in addition a collection of discrete spots. Each odor produced several such spots, generally arranged in a mirrorsymmetric pattern on the two bulbs. Some of these spots appeared in more than one odor image (Fig. 3, arrows), whereas others were activated by just one odor in the series. Qualitatively, it is apparent that each of these pure compounds activates several glomeruli and an individual glomerulus can respond to several odors. Altogether, these odors activated $\sim 40$ glomeruli on the dorsal surface of the two bulbs.

\section{Chemical tuning of glomeruli depends on odor concentration}

For a more quantitative analysis of these relationships, we presented the series of aliphatic aldehydes at four concentrationsspanning three orders of magnitude. The pattern of active glomeruli varied between odorants, but it also depended significantly on their concentration. Figure 4 illustrates the glomerular responses in such an experiment, where 20 spots were observed in a single olfactory bulb. Comparing the patterns of activity elicited by a given odorant at increasing concentrations, one finds that the response amplitude generally increases. In addition, there is a clear change in the response patterns. At low concentrations (dilutions $D=0.0001$ or 0.001 ; see Materials and Methods), each of these odors can be identified by just one or a few specific glomeruli that respond distinctly. With increasing concentration, many more glomeruli participate in the response with comparable amplitudes. The response patterns can still be distinguished but only by considering the entire ensemble of glomeruli. For exam- ple, to distinguish odors $\mathrm{C} 6, \mathrm{C} 7$, and $\mathrm{C} 8$ at high concentration $(D=0.1)$, it is clearly not sufficient to find the glomerulus with the strongest response. Instead it becomes more revealing to identify which glomeruli in the set are not activated by the odor.

This smearing of response patterns suggests that the individual glomerulus becomes less specific in its response at high odor concentrations. This was explored by analyzing the tuning curve of single glomeruli. Figure $5 a$ plots the response as a function of carbon chain length of the odorant, with a different curve for each concentration. As a rule, the glomerulus responds to a limited and compact range of odors along the chain length axis. Only rarely $(<5 \%$ of glomeruli) did we observe tuning curves with more than one distinct peak. This is consistent with a previous report (Rubin and Katz, 1999) and with the local tuning curves of mitral cells (Imamura et al., 1992). As seen in Figure 5a, at low concentrations these curves are often tuned quite sharply, with only a single odorant or two adjacent ones able to elicit a response. At higher concentration, the curves broaden considerably, and often encompass four or five odors in the series.

To pursue this further, we analyzed the concentration dependence of the response for each individual glomerulus (Fig. $5 b)$.Generally, this relationship followed a sigmoid curve. For a quantitative fit, we used the following simple form:

$$
R=R_{\max } \frac{C}{C+K_{\mathrm{i}}} .
$$

Here $R$ is the optical response amplitude, $R_{\max }$ the maximal response, $C$ the odor concentration, and $K_{i}$ is the half-saturating concentration of that odor. For many glomeruli, this simple model - with very few degrees of freedom - provided a satisfying fit to all the odor-binding curves (Fig. $5 b, c$ ). Note that Equation 1 could be interpreted as describing ligand binding to an olfactory receptor with dissociation constant $K_{i}$. Alternatively, the sigmoid shape might result from saturation of some other component of the signal transduction cascade.

For a given glomerulus in the model of Equation 1, the action of any odorant is fully determined by its parameter $K_{i}$, namely the 


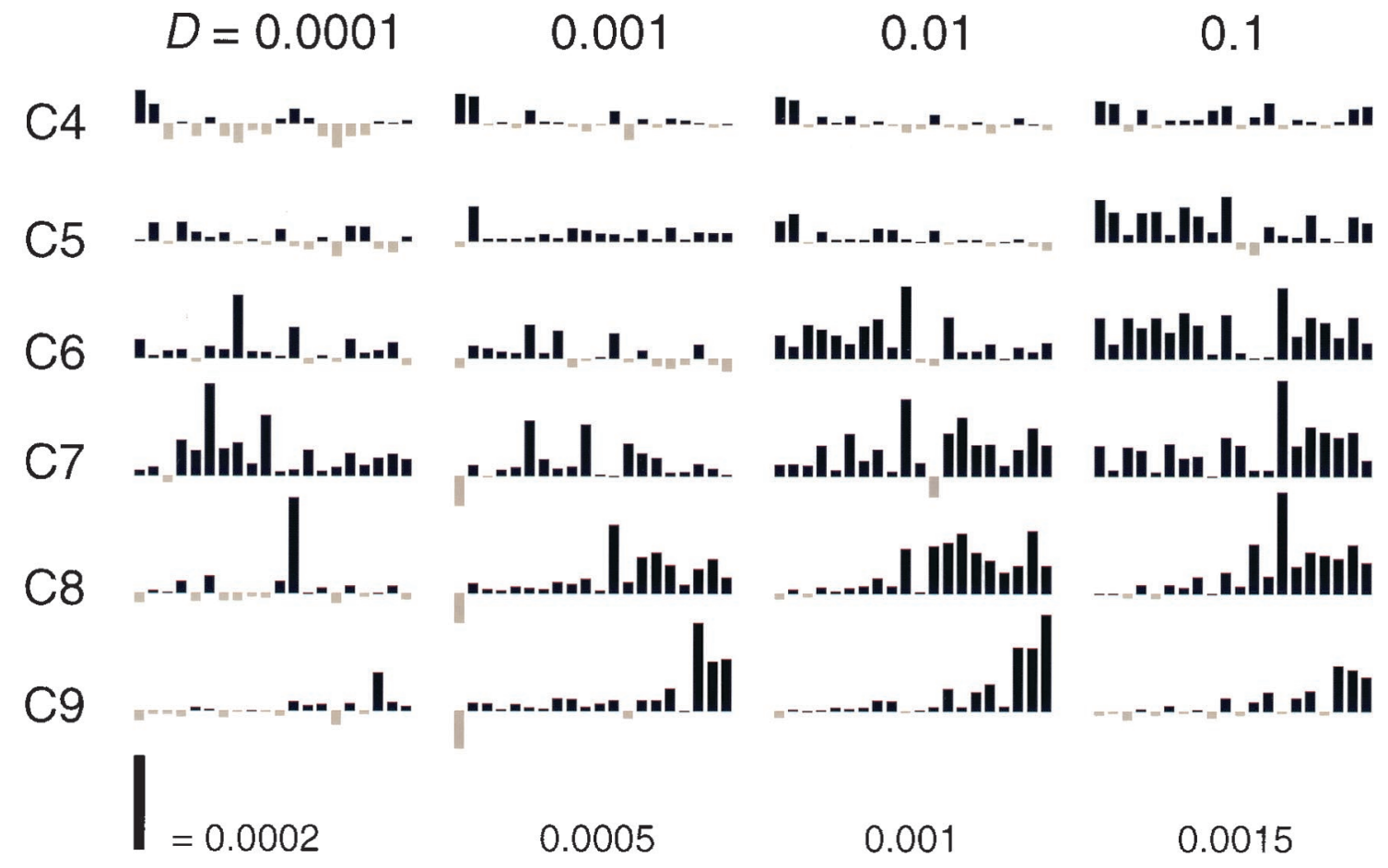

Figure 4. The pattern of activated glomeruli varies with odorant and with concentration. Optical responses of 20 glomeruli in a single olfactory bulb to aliphatic aldehydes of chain length 4 to 9 (C4, ., C9), presented at four different concentrations $(D=0.0001, \ldots, 0.1$, see Materials and Methods for the corresponding vapor concentrations). Each bar graph plots the absorption increase (black) or decrease (gray) for the 20 glomeruli. To better reveal the patterns at low concentrations, the vertical axis was normalized for each of the four concentrations, and the scale for the absorption change is indicated below each column of plots.

concentration that elicits a half-maximal response. We define the "sensitivity" of the glomerulus to an odorant as the inverse of this concentration, and that quantity is plotted as a function of carbon chain length in Figure $5 d$. These curves are generally rather sharp, with significant sensitivity to just one or two adjacent chain lengths. The decrease in sensitivity on either side of the maximum is steep: on average, one $\mathrm{CH}_{2}$ group more or less produces a 10 -fold change in the effective affinity. These sensitivity curves (Fig. $5 d$ ) can be seen as the fundamental chemical spectra of glomeruli, because they effectively summarize the response over a wide range of odor concentrations (Fig. $5 b$ ). They tend to have similar shapes as the tuning curves obtained at low concentration (Fig. $5 a ; D=0.001$ ). This is expected from Equation 1, because for $C \ll K_{\mathrm{i}}$, one has $R \propto 1 / K_{\mathrm{i}}$. By contrast, the tuning curves obtained at high concentration (Fig. $5 a ; D=0.1$ ), are often much broader, because the more effective odors cause response saturation and possibly adaptation (at $C>K_{\mathrm{i}}$ ). The same reasoning accounts for the broadening of the population response pattern at high concentrations (Fig. 4).

\section{A map of aliphatic chain length on the olfactory bulb}

As illustrated above, an individual glomerulus is generally sensitive to one or two adjacent odors in the aliphatic aldehyde series. Thus, each active glomerulus can be characterized by its "preferred chain length", which we computed as the odor chain length weighted by the tuning curve (see Materials and Methods). To illustrate how this property is distributed on the bulb, we marked the location of each glomerulus and color-coded them according to their preferred chain length (Fig. 6). The resulting spatial maps were similar across individuals but not identical in detail. It was not possible to make a clear correspondence between single glomeruli in different animals. However, several intriguing features of the odor maps were found reliably in every individual.

First, the aliphatic aldehydes activated glomeruli only in a restricted portion of the dorsal olfactory bulb, amounting to $\sim 25 \%$ of the visible surface. Within this region, activation was not complete: less than half of the $\sim 50$ glomeruli there showed significant optical responses to an odorant in the aldehyde series.

The maps in the two bulbs were highly mirror-symmetric across the midline. On a coarse scale, this was true for the general region of activation. On a fine scale, one could sometimes pair individual glomeruli in the two bulbs, for example two glomeruli tuned to short chains near the middle of each bulb, and two glomeruli tuned to $\mathrm{C} 9$ at the lateral edges (Fig. 3). Earlier studies had noted another axis of symmetry within each bulb, running posteriorlateral to anterior-medial. This is apparent in the expression patterns for specific olfactory receptors (Ressler et al., 1994; Vassar et al., 1994; Mombaerts, 1999) and in odor maps measured by 2-deoxyglucose uptake (Stewart et al., 1979; Johnson et al., 1999). Such a symmetry axis is visible in some of our maps of the dorsal bulb (Fig. $6 c$, dashed lines) but was difficult to detect in some other rats (Fig. $6 a, b$ ), probably because the axis runs close to the midline, and half of the mirror pattern is hidden on the lateral wall of the bulb.

Within the map, there was a systematic spatial progression of response properties. Near the middle of each bulb, glomeruli were tuned to short chain lengths. Moving anterior and lateral from this point, the preferred chain length increased systematically. Glomeruli responding to the longest aldehydes were found near the lateral edge of the observed region. The entire range from four to nine carbons was mapped onto a span of $\sim 1.5 \mathrm{~mm}$ on the olfactory bulb. This suggests that there is an organized map of 

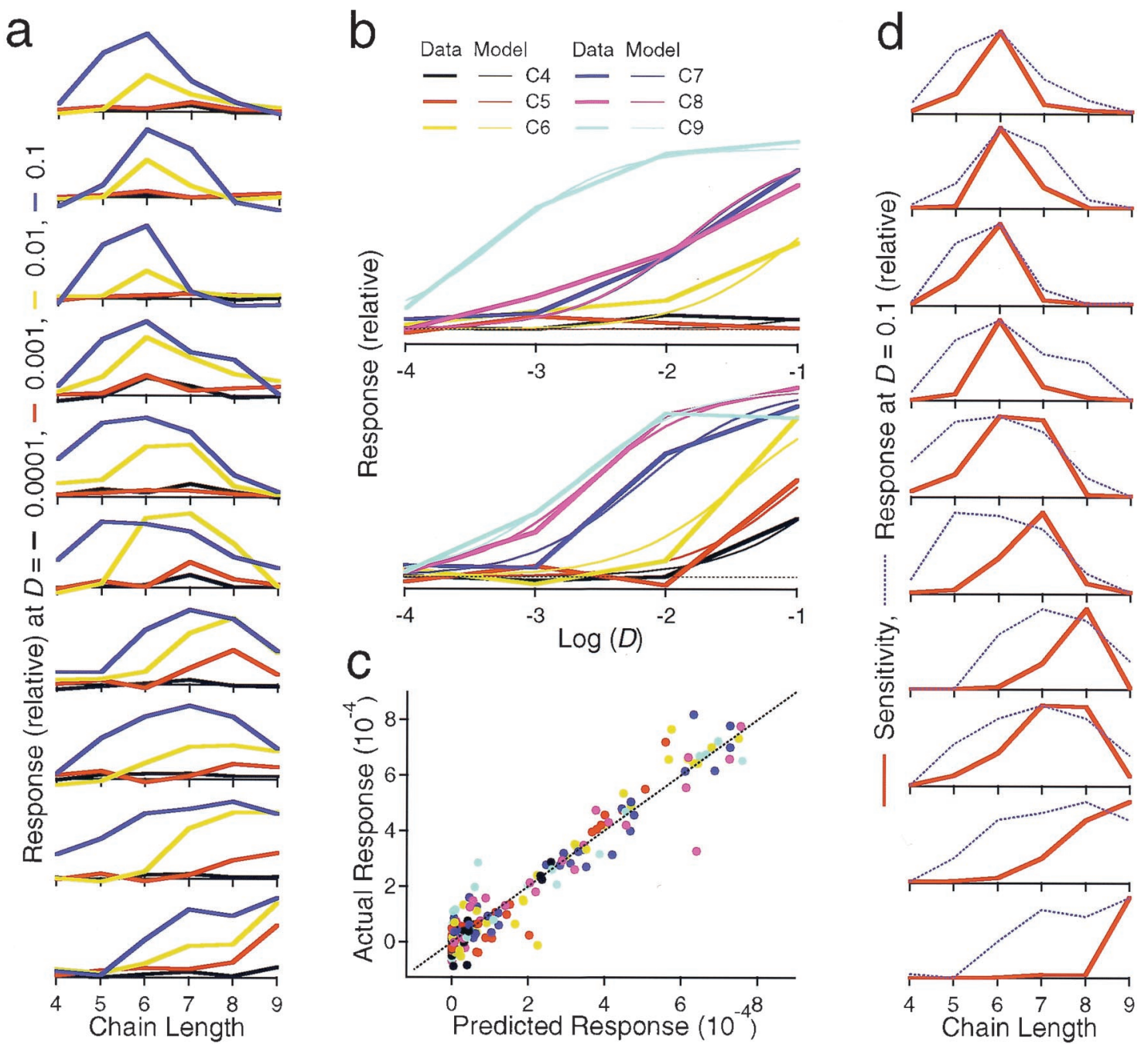

Figure 5. Chemical tuning of individual glomeruli to aliphatic aldehydes. $a$, Optical response plotted as a function of the carbon chain length of the odorant. Each plot represents one of the glomeruli of Figure 4 and contains four curves measured at increasing odor concentrations $D=0.0001,0.001$, 0.01, and 0.1. The vertical axis is scaled for each glomerulus to the maximum response observed. $b$, Response as a function of odor concentration, $D$, for two of the glomeruli in a. Bold lines are measurements for the six different odorants, scaled to the maximal response; thin lines are fits of the first-order binding model (Eq. 1) to the data. Note that the model contains only seven parameters for fitting 28 data points. $c$, Comparison of the actual response and the response predicted by Equation 1 for all 10 glomeruli in $a$. Dotted line is the identity. Colors indicate odorants as in $b . d$, Sensitivity (solid line) plotted as a function of the odorant's carbon chain length, for each of the glomeruli in $a$. Sensitivity is the reciprocal of the half-saturating concentration, determined from the fits of Equation 1 in $b$. Shown for comparison is the response measured at high concentration $(D=0.1$, from $a)$, normalized to the same maximum value (dotted line).

this rather simple stimulus variable-molecular size-on the olfactory bulb, an issue that is pursued below in greater detail.

\section{DISCUSSION}

\section{Topography and local processing in the olfactory bulb}

The choice of stimuli in the present experiments was motivated by several insightful studies of mitral cell responses in rabbit olfactory bulb. Using a series of aliphatic molecules with the same functional group but different chain length, Mori et al. (Imamura et al., 1992; Mori and Yoshihara, 1995; Yokoi et al., 1995) showed that individual mitral cells have a restricted excitatory "receptive range". Only approximately two adjacent molecules in the series are able to excite the neuron. In addition, molecules immediately adjacent to this excitatory range have a pronounced inhibitory effect. Molecules with much shorter or much longer chains produce no response. Along this "chain-length axis" of odor space, the mitral cell therefore has a receptive field similar to that of neurons in the early visual system, with an excitatory "center" and nearby antagonistic "surround." It was proposed that the narrow excitatory range is mediated by the glomerulus in which the mitral cell has its primary dendrite, whereas the adjacent inhibitory range reflects inhibitory inputs from neighboring glomeruli. These local lateral connections could be made by periglomerular cells or by granule cells, although Yokoi et al. (1995) favor the latter alternative.

This hypothesis makes an important prediction: glomeruli with similar preferred chain length must be located near one another, whereas glomeruli with different preferred chain length should be far apart. In this way, local inhibitory circuits will produce a restricted inhibitory surround to the receptive range of the mitral cell. If, instead, glomeruli were arranged with no particular spatial order, then lateral inhibition in space would produce uniform inhibition along the chain length axis. All molecules in the series should then inhibit the response of the mitral cell, counter to what is observed (Yokoi et al., 1995). The electrical recordings showed that neurons sensitive to these aliphatic molecules were concen- 


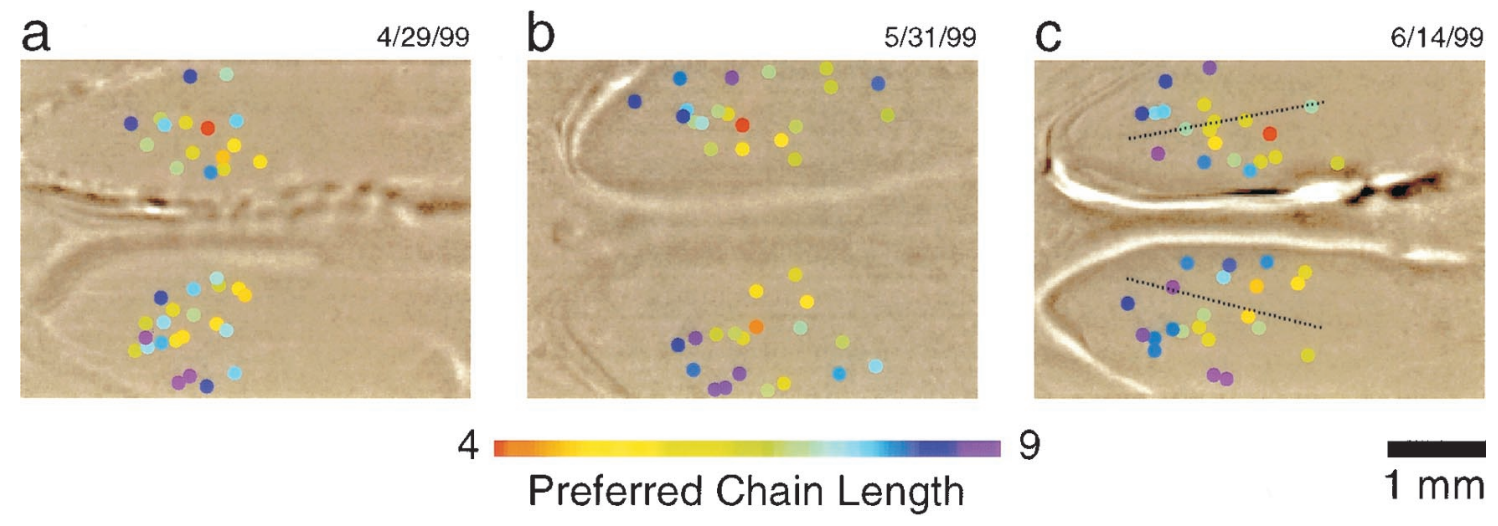

Figure 6. Response maps for aliphatic aldehydes. $a-c$, Results from three different rats. Each panel shows a faint image of the olfactory bulbs, along with the glomeruli responsive to this odor series. Each active glomerulus is marked with a circle and colored according to its preferred chain length. The dotted lines in $c$ indicate a suggested line of symmetry within the bulb.

trated in a small region of the dorsomedial bulb. Within that region "neurons with different tuning specificities were found to be intermingled" (Imamura et al., 1992), although the spatial sampling achieved by these electrical recordings was somewhat sparse.

Inspection of Figure 6 shows that the rat, like the rabbit, has a subregion of the dorsal bulb sensitive to aliphatic aldehydes. More importantly, there is in fact a systematic map of odorant chain length within this region. The preferred chain length of the glomeruli increases gradually from the middle of the bulb outward in the rostral and lateral direction. There may also be a symmetric pattern on the medial side (Fig. $6 c$ ) that extends down the medial wall and is partly obscured from dorsal view. Based on measurements of 2-deoxyglucose uptake, Johnson et al. (1999) reported that the odor images of aliphatic acids gradually shift rostral with increasing carbon chain length. Because the detailed layout of glomeruli varies across animals (compare the three panels in Fig. 6), the odor maps measured by that method have lower spatial resolution (Johnson et al., 1998), such that the entire region activated in Figure 6 would likely produce a single "uptake field." The center of that field would indeed shift rostral for longer chain lengths. However, with the glomerular resolution afforded by optical imaging we found that activation within such a field of related glomeruli is not contiguous and that the region contains many glomeruli that do not respond to the aldehyde series.

As a result of the systematic progression of response properties on the bulb, nearby glomeruli tend to have similar tuning for carbon chain length. Figure 7 demonstrates this explicitly. When two glomeruli were separated by $<200 \mu \mathrm{m}$, they had similar preferred chain lengths, whereas two distant glomeruli in the set exhibited rather different chemical tuning. Thus, the odor map on the olfactory bulb does indeed have a fine-grained topography on the scale of a few glomerular diameters. Therefore, local inhibitory circuits in the olfactory bulb could produce the observed "center-surround" receptive fields along the chain length axis.

Whereas this may be a strategy to sharpen the response of the individual mitral cell (Yokoi et al., 1995), making it more discriminative among molecules with only slightly different properties, an alternative interpretation is that lateral inhibition serves to "decorrelate" the signals from similar olfactory receptors. The afferent input to two neighboring glomeruli may be highly correlated in the course of natural experience, either because their chemical spectra overlap to include some of the same compounds or because their preferred ligands tend to occur together in the same odor source. Under these conditions it is redundant to represent two very similar signals twice, and a more efficient strategy is to compute and analyze their difference. This could be achieved by lateral inhibitory connections between the appropriate glomeruli. The analogous situation occurs in vision, in which the signals of nearby photoreceptors are highly correlated simply because the natural world is made of objects with uniform intensity over extended regions. Lateral inhibition in the retina serves to decorrelate the raw visual image and produces a more compact representation suited to further processing (Atick and Redlich, 1992; Van Hateren, 1993).

In contrast to the visual system, in which photoreceptors with nearby receptive fields are by design located close to each other in the retina, the olfactory system must explicitly construct a map appropriate for local interactions, by routing the olfactory receptor axons to the appropriate bulb location. There appears to be a serious limit to this mapping strategy in the fact that the olfactory bulb surface has only two dimensions. This means that at most two stimulus properties could be mapped continuously onto the two spatial variables. Yet, one imagines that there are many properties of olfactant molecules other than chain length that are analyzed and compared by local bulb circuits. A similar dilemma is encountered by the primary visual cortex. The two dimensions of this structure are needed to map the two dimensions of visual field location. Yet the cortex is also interested in other stimulus variables, such as the eye of origin and the orientation of local edges. These stimulus properties are represented in systematic maps that are discontinuous and intercalated into the global spatial map on a finer scale (Hübener et al., 1997). In this context, it is important to note that the glomeruli activated by aldehydes are interspersed by many others that did not respond to this chemical series (Fig. 6). A broad survey with many olfactants might reveal maps of other molecular properties intercalated on the surface of the bulb. Because the spatial layout of these signals affects how they can be combined by local circuitry, further analysis of this topography can help us understand the nature of neural computations in the olfactory bulb.

\section{The chemical spectra of olfactory receptors}

Over the past few years, a simple hypothesis has emerged for how olfactory bulb glomeruli are connected to the sensory periphery, summarized as "one glomerulus-one receptor." It is thought that each glomerulus receives inputs from just one type of sensory neuron and that each sensory cell expresses just one type of olfactory receptor (Mori et al., 1998). In this picture, the glomerulus-more specifically its afferent input signal-interacts with the world of volatile chemicals through just one chemical binding 

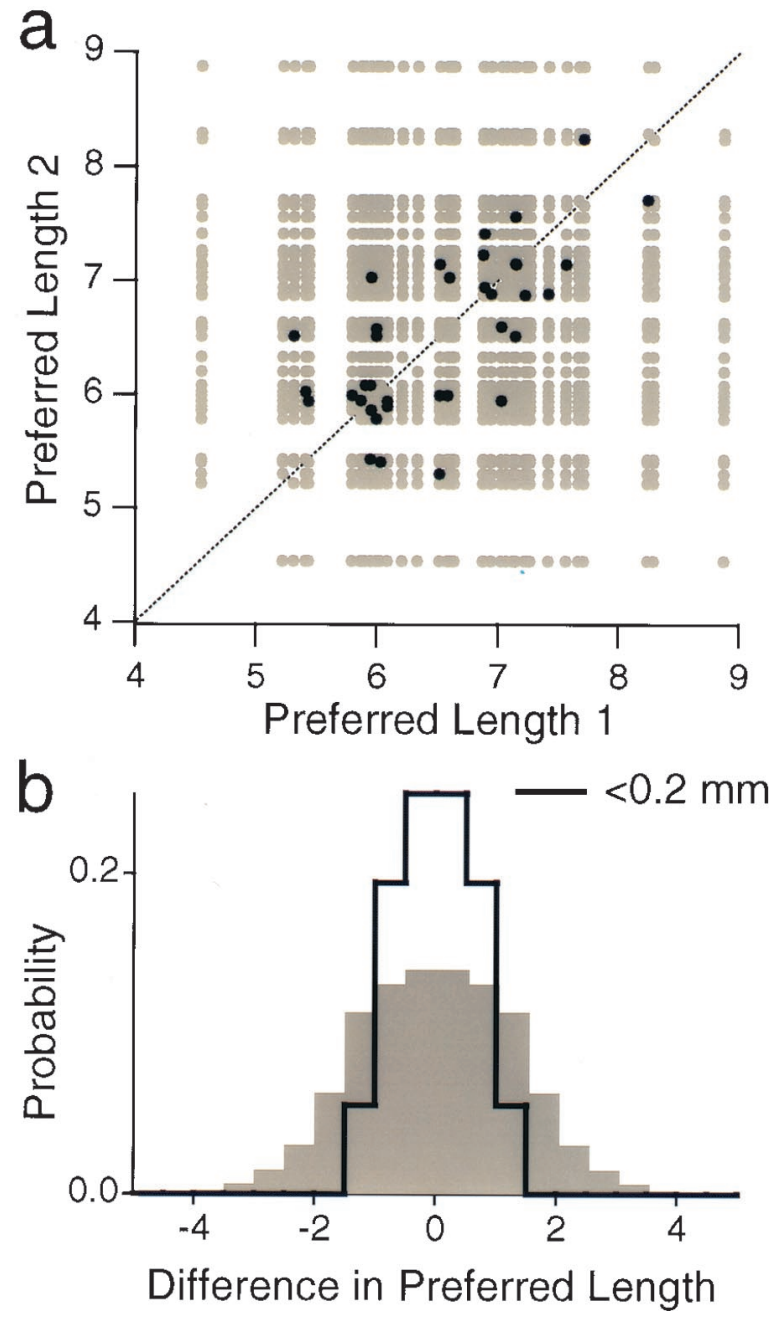

Figure 7. Nearby glomeruli have similar chemical tuning. $a$, A scatterplot of preferred chain lengths for each member of a pair of glomeruli in the map of Figure $6 c$. The gray dots compare every glomerulus with every other, whereas the black dots represent pairs of glomeruli located within $0.2 \mathrm{~mm}$ of each other. Note that the black dots tend to fall close to the identity (dotted line). $b$, The results of $a$ were projected along the diagonal, producing a histogram of the difference in preferred chain length between two glomeruli. The gray plot includes all pairwise comparisons; the black line only those within $0.2 \mathrm{~mm}$ of each other. Again, local comparisons are seen to produce smaller differences in chemical tuning.

site. Different odors will bind to this site with different affinity, but once the receptor is activated, the glomerular response is independent of the identity of the ligand. A low concentration of high-affinity ligand produces the same effects as a suitably higher concentration of low-affinity ligand. This is analogous to the "principle of univariance" in vision. A photoreceptor produces exactly the same response to two lights of different wavelengths if their intensities are matched according to the absorption spectrum of the visual pigment (Naka and Rushton, 1966). Therefore, the concentration-response curves for different odors (Fig. 5b) should all have the same shape when plotted on a logarithmic concentration axis, only different lateral offsets. This is a fairly strong prediction, because it holds not only for the steady-state activity measured here, but for all temporal aspects of the response. It is predicated only on the assumption of a single odorantbinding site. For the glomeruli activated in this study, this univariance was in fact observed (Fig. 5b,c). In contrast, many postsynaptic neurons in the olfactory bulb-mitral cells, tufted cells, and interneurons-do not obey univariance. When testing the same cells with different odorants, one often finds concentration-response curves of very different shape (Wellis et al., 1989). One can immediately conclude that these neurons must receive odor information through more than one type of receptor binding site. For example, although the mitral cell extends its primary dendrite into a single glomerulus, it also receives signals from other glomeruli via interneurons synapsing on its secondary dendrites. The uptake of 2-deoxyglucose in the glomerular layer of the olfactory bulb has also been reported to follow a different concentration dependence for different odors (Johnson and Leon, 2000). However, the "uptake fields" identified in these experiments typically pool the activity of a handful of glomeruli (Johnson et al., 1998). Thus, the assumption of a single receptor binding site is violated, and one would expect deviations from univariance.

The univariance of the intrinsic signals measured here could be explained if they derive primarily from the axon terminals of receptor cells, rather than postsynaptic neurons. To the extent that "one glomerulus-one receptor" is correct, the optical responses then reflect ligand binding to the corresponding receptor molecule. One can compare the efficacy with which different ligands bind the receptor by determining the concentration that produces a half-saturated response ( $K_{i}$ in Eq. 1). The resulting curve of sensitivity as a function of odor (Fig. $5 d$ ) is the precise analog of a visual absorption spectrum as a function of wavelength, which can be derived from half-saturated responses of a photoreceptor (Baylor and Hodgkin, 1973). For the present series of aldehydes, the sensitivity curves (Fig. $5 d$ ) often had rather sharp peaks, indicating a 10 -fold change in binding affinity when the ligand was lengthened or shortened by just one $\mathrm{CH}_{2}$ group. Of course, these same glomeruli may well respond to other aliphatic molecules, possibly with a similar chain length tuning (Imamura et al., 1992).

A more conventional way to measure the tuning curve of a receptor has been to present all odors at one concentration and to simply plot the response as a function of odor. Based on this, a number of studies have concluded that individual olfactory neurons respond to a very broad range of chemicals. Almost invariably, these experiments used a very high concentration, typically $10-40 \%$ of the saturated vapor (Duchamp et al., 1974; Revial et al., 1978; Revial et al., 1982; Sicard and Holley, 1984; Guthrie and Gall, 1995; Duchamp-Viret et al., 1999), with the occasional puff of fully saturated vapor directly onto the mucosa (Mathews, 1972). In nature, sniffs of saturated vapor occur infrequently, except for the rare occasion when an animal needs to swim across a pool of amyl acetate. Instead, rats are found to detect and discriminate odors at $10^{-6}$ of the saturated vapor (Passe and Walker, 1985), and given the difficulties of behavioral measurements, this is probably a conservative estimate. In our sample, the most sensitive glomeruli gave a half-maximal response to their preferred odorant already at 0.0002 of the saturated vapor concentration, and all glomeruli tested had a half-maximal response at $<0.01$ of saturated vapor. When we measured tuning curves at higher concentration, although still far from saturated vapor, they were significantly broader than the affinity spectra (Fig. $5 d$ ). This results simply because the olfactory response is compressed by saturation (Eq. 1), such that high-affinity and low-affinity ligands have the same effect.

For the same reasons of saturation, a given odorant, when used at high concentration, produces a broad activity pattern in the field of glomeruli (Fig. 4) because it now activates also those receptors for which it is not the optimal ligand. When the concentration is reduced, the active population contracts, as has been 
observed consistently for many odors and species (Stewart et al., 1979; Duchamp-Viret et al., 1989; Imamura et al., 1992; Sato et al., 1994; Cinelli et al., 1995; Guthrie and Gall, 1995; Friedrich and Korsching, 1997; Johnson et al., 1999; Rubin and Katz, 1999). Both the sensory neurons (Firestein et al., 1993) and the glomeruli (Johnson and Leon, 2000) (Fig. 5b) have a sharp dependence of response on concentration, so that individual glomeruli drop out of the odor image rather suddenly as the concentration decreases. The effects can be dramatic, at times a twofold reduction in the number of active receptor types for a twofold decrease in concentration (Revial et al., 1982). If one extrapolates this behavior to concentrations several orders of magnitude lower, as might be encountered under natural conditions, it is plausible that only one or a few glomeruli in the entire bulb remain activated to any significant degree. In fact, frustration by the scarcity of single-neuron responses is the prime motivation for using abnormally high odor concentrations (Revial et al., 1978). Thus, one should at least bear in mind the possibility that the real-life neural code for odors is sparse, rather than highly combinatorial, with only a few glomeruli active at any one time.

\section{REFERENCES}

Atick JJ, Redlich AN (1992) What does the retina know about natural scenes? Neural Comput 4:196-210.

Baylor DA, Hodgkin AL (1973) Detection and resolution of visual stimuli by turtle photoreceptors. J Physiol (Lond) 234:163-198.

Bonhoeffer T, Grinvald A (1996) Optical imaging based on intrinsic signals: the methodology. In: Brain mapping: the methods (Toga AW, Mazziotta JC, eds), pp 55-97. London: Academic.

Buck LB (1996) Information coding in the vertebrate olfactory system. Annu Rev Neurosci 19:517-544.

Buck L, Axel R (1991) A novel multigene family may encode odorant receptors: a molecular basis for odor recognition. Cell 65:175-187.

Cinelli AR, Hamilton KA, Kauer JS (1995) Salamander olfactory bulb neuronal activity observed by video rate, voltage-sensitive dye imaging. III. Spatial and temporal properties of responses evoked by odorant stimulation. J Neurophysiol 73:2053-2071.

Duchamp A, Revial MF, Holley A, MacLeod P (1974) Odor discrimination by frog olfactory receptors. Chem Senses 1:213-233.

Duchamp-Viret P, Duchamp A, Vigouroux M (1989) Amplifying role of convergence in olfactory system a comparative study of receptor cell and second-order neuron sensitivities. J Neurophysiol 61:1085-1094.

Duchamp-Viret P, Chaput MA, Duchamp A (1999) Odor response properties of rat olfactory receptor neurons. Science 284:2171-2174.

Firestein S, Picco C, Menini A (1993) The relation between stimulus and response in olfactory receptor cells of the tiger salamander. J Physiol (Lond) 468:1-10.

Friedrich RW, Korsching SI (1997) Combinatorial and chemotopic odorant coding in the zebrafish olfactory bulb visualized by optical imaging. Neuron 18:737-752.

Gesteland RC, Lettvin JY, Pitts WH, Rojas A (1963) Odor specificities of the frog's olfactory receptors. In: Olfaction and taste, Wenner-Gren Center international symposium series, Vol 1 (Zotterman Y, ed), pp 19-34. Oxford, NY: Pergamon.

Guthrie KM, Gall CM (1995) Functional mapping of odor-activated neurons in the olfactory bulb. Chem Senses 20:271-282.

Hübener M, Shoham D, Grinvald A, Bonhoeffer T (1997) Spatial relationships among three columnar systems in cat area 17. J Neurosci 17:9270-9284.

Imamura K, Mataga N, Mori K (1992) Coding of odor molecules by mitral/tufted cells in rabbit olfactory bulb. I. Aliphatic compounds. J Neurophysiol 68:1986-2002.

Johnson BA, Leon M (2000) Modular representations of odorants in the glomerular layer of the rat olfactory bulb and the effects of stimulus concentration. J Comp Neurol 422:496-509.

Johnson BA, Woo CC, Leon M (1998) Spatial coding of odorant features in the glomerular layer of the rat olfactory bulb. J Comp Neurol 393:457-471.

Johnson BA, Woo CC, Hingco EE, Pham KL, Leon M (1999) Multidimensional chemotopic responses to $N$-aliphatic acid odorants in the rat olfactory bulb. J Comp Neurol 409:529-548.

Jourdan F, Duveau A, Astic L, Holley A (1980) Spatial distribution of [14C]2-deoxyglucose uptake in the olfactory bulbs of rats stimulated with two different odours. Brain Res 188:139-154.

Katoh K, Koshimoto H, Tani A, Mori K (1993) Coding of odor mole- cules by mitral/tufted cells in rabbit olfactory bulb. II. Aromatic compounds. J Neurophysiol 70:2161-2175.

Laurent G (1999) A systems perspective on early olfactory coding. Science 286:723-728.

Mathews DF (1972) Response patterns of single units in the olfactory bulb of the rat to odor. Brain Res 47:389-400.

Mayhew JE, Askew S, Zheng Y, Porrill J, Westby GW, Redgrave P, Rector DM, Harper RM (1996) Cerebral vasomotion: a 0.1-Hz oscillation in reflected light imaging of neural activity. NeuroImage 4:183-193.

Mayhew J, Zheng Y, Hou Y, Vuksanovic B, Berwick J, Askew S, Coffey $\mathrm{P}$ (1999) Spectroscopic analysis of changes in remitted illumination: the response to increased neural activity in brain. NeuroImage 10:304-326.

Meisami E, Sendera TJ (1993) Morphometry of rat olfactory bulbs stained for cytochrome oxidase reveals that the entire population of glomeruli forms early in the neonatal period. Brain Res Dev Brain Res $71: 253-257$.

Mombaerts P (1999) Molecular biology of odorant receptors in vertebrates. Annu Rev Neurosci 22:487-509.

Mombaerts P, Wang F, Dulac C, Chao SK, Nemes A, Mendelsohn M, Edmondson J, Axel R (1996a) Visualizing an olfactory sensory map. Cell 87:675-686.

Mombaerts P, Wang F, Dulac C, Vassar R, Chao SK, Nemes A, Mendelsohn M, Edmondson J, Axel R (1996b) The molecular biology of olfactory perception. Cold Spring Harb Symp Quant Biol 61:135-145.

Mori K, Yoshihara Y (1995) Molecular recognition and olfactory processing in the mammalian olfactory system. Prog Neurobiol 45:585-619.

Mori K, Nagao H, Sasaki YF (1998) Computation of molecular information in mammalian olfactory systems. Network 9:R79-R102.

Mori K, Nagao H, Yoshihara Y (1999) The olfactory bulb: coding and processing of odor molecule information. Science 286:711-715.

Naka KI, Rushton WA (1966) S-potentials from colour units in the retina of fish (Cyprinidae). J Physiol (Lond) 185:536-555.

Passe DH, Walker JC (1985) Odor psychophysics in vertebrates. Neurosci Biobehav Rev 9:431-467.

Ressler KJ, Sullivan SL, Buck LB (1994) Information coding in the olfactory system: evidence for a stereotyped and highly organized epitope map in the olfactory bulb. Cell 79:1245-1255.

Revial MF, Duchamp A, Holley A (1978) Odour discrimination by frog olfactory receptors: a second study. Chem Senses 3:7-21.

Revial MF, Sicard G, Duchamp A, Holley A (1982) New studies on odour discrimination in the frog's olfactory receptor cells. I. Experimental results. Chem Senses 7:175-190.

Rubin BD, Katz LC (1999) Optical imaging of odorant representations in the mammalian olfactory bulb. Neuron 23:499-511.

Sato T, Hirono J, Tonoike M, Takebayashi M (1994) Tuning specificities to aliphatic odorants in mouse olfactory receptor neurons and their local distribution. J Neurophysiol 72:2980-2989.

Shepherd GM (1994) Discrimination of molecular signals by the olfactory receptor neuron. Neuron 13:771-790.

Sicard G, Holley A (1984) Receptor cell responses to odorants: similarities and differences among odorants. Brain Res 292:283-296.

Stewart WB, Kauer JS, Shepherd GM (1979) Functional organization of rat olfactory bulb analysed by the 2-deoxyglucose method. J Comp Neurol 185:715-734

Takatani S, Graham MD (1979) Theoretical analysis of diffuse reflectance from a two-layer tissue model. IEEE Trans Biomed Eng 26:656-664.

Uchida N, Takahashi YK, Tanifuji M, Mori K (2000) Odor maps in the mammalian olfactory bulb: domain organization and odorant structural features. Nat Neurosci 3:1035-1043.

Van Hateren JH (1993) Spatiotemporal contrast sensitivity of early vision. Vision Res 33:257-267.

Vanzetta I, Grinvald A (1999) Increased cortical oxidative metabolism due to sensory stimulation: implications for functional brain imaging. Science 286:1555-1558.

Vassar R, Chao SK, Sitcheran R, Nunez JM, Vosshall LB, Axel R (1994) Topographic organization of sensory projections to the olfactory bulb. Cell 79:981-991.

Wellis DP, Scott JW, Harrison TA (1989) Discrimination among odorants by single neurons of the rat olfactory bulb. J Neurophysiol 61:1161-1177.

Wong-Riley M (1979) Changes in the visual system of monocularly sutured or enucleated cats demonstrable with cytochrome oxidase histochemistry. Brain Res 171:11-28.

$\mathrm{Xu} \mathrm{F}$, Greer CA, Shepherd GM (2000) Odor maps in the olfactory bulb. J Comp Neurol 422:489-495.

Yokoi M, Mori K, Nakanishi S (1995) Refinement of odor molecule tuning by dendrodendritic synaptic inhibition in the olfactory bulb. Proc Natl Acad Sci USA 92:3371-3375. 